\title{
Racial Disparities in Parkinson Disease: A Systematic Review of the Literature
}

\author{
Chantale 0. Branson, Andrew Ferree, Anna D. Hohler, Marie-Helene Saint-Hilaire \\ Department of Neurology, Boston University School of Medicine, Boston, MA, USA \\ Email: cmurray@bu.edu, andrew.ferree@gmail.com, Anna.Hohler@bmc.org, Marie.Saint-Hilaire@bmc.org
}

How to cite this paper: Branson, C.O., Ferree, A., Hohler, A.D. and Saint-Hilaire, M.-H. (2016) Racial Disparities in Parkinson Disease: A Systematic Review of the Literature. Advances in Parkinson's Disease, 5, 87-96.

http://dx.doi.org/10.4236/apd.2016.54011

Received: October 27, 2016

Accepted: November 21, 2016

Published: November 25, 2016

Copyright $\odot 2016$ by authors and Scientific Research Publishing Inc. This work is licensed under the Creative Commons Attribution International License (CC BY 4.0).

http://creativecommons.org/licenses/by/4.0/

(c) (i) Open Access

\begin{abstract}
Racial differences in the prevalence of Parkinson's disease (PD) have been reported for decades. Many of the earliest reports were flawed because they were based on crude datasets, such as hospital databases, death certificates, door-to-door surveys and records of Medicare beneficiaries. These studies provided conflicting results and were found to have numerous biases. Publications with improved study designs in recent years have yielded higher quality findings that are worth reviewing. We reviewed studies published between 2005 and 2014 that analyzed the racial differences in Parkinson's disease diagnosis, treatment-including deep brain stimulation-and access to care. Literature searches were conducted in PubMed and EBSCO. These studies highlight advances in the field and explore differences in PD among ethnic and racial groups. Our literature review focused on prevalence, treatment and diagnosis discrepancies, and racial variations in the perceptions of aging. An appraisal of twelve reviewed studies determined a decrease in prevalence and incidence of PD in Americans of African descent compared to Caucasians. The studies also showed multiple health disparities, including lack of access to care, treatment, and inclusion in research. More studies are needed to address the causes and prevention of health disparities, as well as solutions, such as community outreach.
\end{abstract}

\section{Keywords}

Racial and Ethnic Disparities, Epidemiology, Parkinson's Disease

\section{Introduction}

Parkinson's disease (PD) is a neurodegenerative disorder that is diagnosed based on clinical features, including bradykinesia, resting tremor, rigidity, and postural stability. These clinical findings are essential for physicians to make an accurate diagnosis, which subsequently will lead to improvement of clinical outcomes and quality of care. Studies 
have suggested there is a lower prevalence of PD among African-Americans. It is unclear if this is due to genetics, or inequalities among access to care, diagnosis, and treatment.

In 2004, McInerney-Leo et al. analyzed prevalence and incidence of PD among African-Americans compared to Caucasians [1]. There were six studies that analyzed the prevalence and incidence of PD through hospital records, physician registration and death certificates. The results were conflicting because of biased study designs, including low power, healthcare access, physician bias and ascertainment bias. The article recommended future studies conduct a long-term survey of the population to determine the incidence and phenotype of PD among Caucasians compared to African-Americans.

Understanding the epidemiological characters of PD among minorities will help to address the disparity in the care of this population. It will also ultimately provide a deeper understanding of the biological, environmental, and genetic mechanisms of the disease. Therefore, we undertook a literature review of racial disparities in PD focusing on articles published since 2004 .

\section{Methods}

We performed a database search of articles published after 2004 that studied racial disparities in every facet of Parkinson's disease among patients of African ancestry compared to Caucasians. Topics included access to care, diagnosis, treatment, and inclusion in research. A search in PubMed and Medline of literature with the following terms: (racial disparities and Parkinson's disease) and (racial disparities and veterans affairs) resulted in ten studies; Two additional articles were obtained from references of the studies elicited from PubMed and Medline. We reviewed twelve studies published between 2005 and 2014, which are found in Table 1. Many of the studies used the term white for Caucasian and black for African-American. A summation of the studies including the study design, study type, results, limitations and strengths are discussed below.

\section{Results}

\subsection{Studies at Tertiary Movement Disorders Centers}

In 2011, Hemming et al. assessed the racial and socioeconomic disparities (annual income and educational level) via cross-sectional analysis of patients seen at a tertiary Movement Disorders center at the University of Maryland. The authors evaluated the prevalence of PD among patients who self-identified as African-Americans compared to whites (93.4\% white and 6.1\% African-American).

The study revealed that African-Americans, at the time of diagnosis, had greater disability, disease severity, and were prescribed fewer anti-parkinson medications than Caucasians. Most notably, African-Americans had a two-fold increase in years between disease onset and diagnosis [2]. The limitations of this study included a small sample size (66 African-Americans) and referral bias given a select population referred to a tertiary center. Other confounding factors, such as socioeconomic status including income 
Table 1. Twelve studies analyzing the prevalence, incidence, management and treatment of PD among whites compared with non-whites.

\begin{tabular}{llll}
\hline Author \& Journal Studies & Study Sample Comment
\end{tabular}

Pressley et al. 2005

Movement Disorders

Disparities in the Recording of PD on Death Certificates

Cheng et al. 2008

Parkinsonism and

Related Disorders

Dahodwala et al. 2009

Annals of Neurology

Dahodwala et al. 2009

Movement Disorders

Willis et al. 2009

Neuroepidemiology

Yacoubian et al. 2009

Neuroepidemiology

Dahodwala et al. 2011

Neuroepidemiology

Hemming et al. 2011

Archives Neurology

Dahodwala et al.

2012 PLOS ONE

Tilley et al. 2012

Clinical Trials

Movement (ERM)
Disparities of care in veterans with Parkinson's

Treatment disparities in Parkinson's Disease

Racial Differences in the Diagnosis of Parkinson's Disease

Geographic and Ethnic Variation in Parkinson Disease: A Population-Based Study of US Medicare Beneficiaries

Racial Differences in Parkinson's Disease Medication Use in the Reasons for Geographic and Racial Differences in Stroke Cohort: A Cross-Sectional Study

Delayed Parkinson's Disease Diagnosis among African-Americans: The role of reporting of disability

Racial and Socioeconomic Disparities

in Parkinsonism

Validation of an Instrument to

Measure Older Adults'

Expectations Regarding

A randomized recruitment intervention trial in Parkinson's disease to increase participant diversity: early stopping for lack of efficacy [15]
Black non-Hispanic 37/751 (4.9\%)

White, non-Hispanic 676/751 (90.0\%)

Hispanic/other 38/751 (5.1\%)

Non-Hispanic White

$\mathrm{N}=309 / 374(82.62 \%)$

African-American $\mathrm{N}=65 / 374$ (9.35\%)

Hispanic or Latino $\mathrm{N}=30 / 374(8.02 \%)$

Asian $\mathrm{N}=11 / 374$ (2.94\%)

Native American N = 1/375 (0.27\%)

African-American N = 43 (14\%)

White $\mathrm{N}=264(86 \%)$

Non-Hispanic White

$\mathrm{N}=123,489$ (68\%)

Black N = 50,808 (28\%)

Hispanic/Latino $\mathrm{N}=7,974$ (4\%)

White $\mathrm{N}=25,581,561(86.6 \%)$

Black $\mathrm{N}=2,356,271(8.0 \%)$

Hispanic $\mathrm{N}=593,234$ (2.0\%)

Asian $\mathrm{N}=470,024$ (1.6\%)

Native American $\mathrm{N}=96,262(0.3 \%)$

Unknown $\mathrm{N}=66,448(0.2 \%)$

Other $\mathrm{N}=367,034(1.2 \%)$

Black $\mathrm{N}=10,123(41.7 \%)$

White $\mathrm{N}=14,242(58.3 \%)$

African-American $\mathrm{N}=16$ (22\%)

White $\mathrm{N}=58(78 \%)$

Total population $\mathrm{N}=1090$

African-American $\mathrm{N}=66$ (6.05\%)

White $\mathrm{N}=1024$ (93.94\%)

Total population $\mathrm{N}=192$

African-American N = 60 (31\%)

White $\mathrm{N}=119(62 \%)$

Latino $\mathrm{N}=3(2 \%)$

Caucasian $\mathrm{N}=1572(90.2 \%)$

African-American N = 40 (2.3\%)

Hispanic/Latino $\mathrm{N}=75(4.3 \%)$

Asian $\mathrm{N}=42(2.4 \%)$

Other $\mathrm{N}=13(0.8 \%)$
By studying the prevalence of PD based on death certificates the findings showed socioeconomic biases confounding the results.

$6 \% \mathrm{racial} / \mathrm{ethnic}$ disparity of adherence to PD quality indicators among non-Hispanic whites compared to non-whites with PD.

African-American patients did not receive similar treatment for PD compared to white patients.

PD diagnosis among AfricanAmericans was half the rate of whites.

1. The mean prevalence of $P D$ per 100,000 is $1.6 \%$ of the elderly population over 65 years of age.

2. Prevalence in Blacks and Asians was $50 \%$ lower compared to Whites.

3. Blacks have a higher PD related morbidity than whites.

1. Whites more frequently used PD medications compared with blacks.

2. The group without health insurance was less likely to take PD medications.

Newly diagnosed PD among AfricanAmericans were found to have a greater motor impairment compared to whites.

African-Americans were found to have a higher disability and disease severity compared with whites at a tertiary Movement Disorders Center.

Understanding expectations about movement among the elderly through development of a scale.

The Ancillary Trial lacked the overall number of participants that were needed for true analysis and was stopped early. 


\section{Continued}

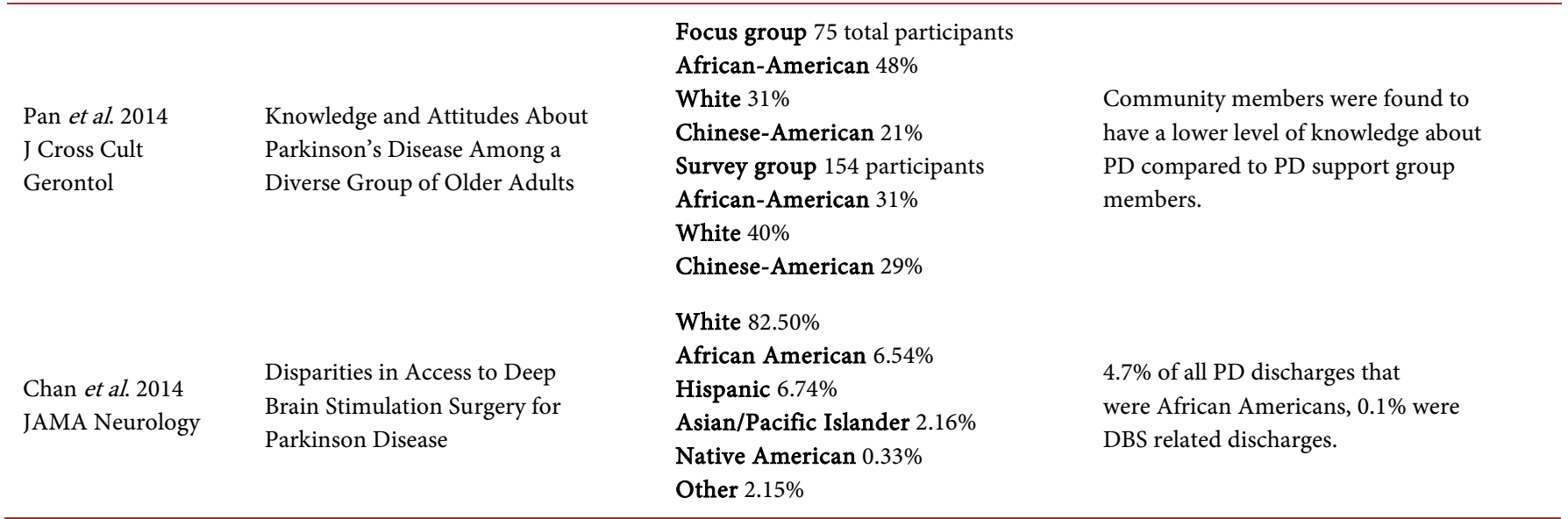

and level of education. The authors stated racial disparities are multi-faceted, and recommend additional studies that assess the attitudes of patients and physicians regarding PD, PD referral and treatment.

\subsection{Studies Using Databases}

Willis et al. assessed racial differences in PD via a cross-sectional study, which also identified potential environmental factors. In this study, PD cases were identified from the United States Medicare benefit recipients with ICD-9 codes 332 or 332.0 in 1995, and from 2000 to 2005. Benefits recipients with ICD-9 codes 332.1 (secondary Parkinsonism) or 333.0 (other degenerative disease of the basal ganglia) were excluded. The sample size was 450,000 PD cases per year. Medicare denominator files included birth, race, sex, residence, zip code, and mortality data for US Americans over the age of 65 . It did not include people who were eligible for Medicare, but were in a health maintenance organization. The mean prevalence of PD was 1,588.43/100,000 among Medicare beneficiaries over age 65 . Of the Medicare recipients, the mean age-related prevalence of PD was 1671.63/100,000 among Caucasian enrollees and 1,036.41/100,000 among African-American enrollees. The mean annual incidence of PD for Medicare recipients from 2002 to 2005 was $445.79 / 100,000$ and this remained stable since 1995 [3].

This was a well-designed study, which was well powered due to the large sample size. It also analyzed the prevalence and incidence of $\mathrm{PD}$, which included demographic as well as geographic factors. Willis et al. confirmed that the prevalence among AfricanAmericans were fifty percent lower than the prevalence among Caucasians. The incidence ratio among African-Americans as compared to Caucasians were higher than the prevalence ratio, suggesting African-Americans have a greater PD-related mortality than Caucasians. The study also reduced referral and selection bias because the cases were obtained from a database. Geographic and demographic variables were analyzed as well, which revealed a higher prevalence of PD in the Midwest and Northeast areas. The regions were termed the "Parkinson disease belt." The limitations of this study included data collection. Obtaining Medicare files excluded patients who were less than 
65 years of age. Although, the incidence of PD increases with age, the mean age of onset is 61.6 years, so patients who are not yet on Medicare were excluded [4]. The use of billing codes can result in false positives due to miscoding of other parkinsonian syndromes as PD. The risk of false negatives may be higher among African-Americans if there is a delay in diagnosing PD. Lack of access to care may exclude African-Americans from obtaining Medicare.

In another study, Dahodwala et al. reviewed Pennsylvania Medicaid claims from January 1, 1999 to December 31, 2003 to evaluate treatment disparities in PD [5]. Cases that included a claim for secondary Parkinsonism, such as schizophrenia and bipolar disorder were excluded. Three hundred seven new cases of PD were identified based on the criteria of Medicaid enrollment, having one Medicaid reimbursement claim associated with $\mathrm{PD}$, and no previous claims associated with PD or Parkinsonism related disorders.

Treatment for PD included medications, physical therapy and/or a return clinic visit for PD within six months of the initial diagnosis. Of the new cases, $86 \%$ were Caucasian and $14 \%$ were African-American. There were no statistical differences in age, sex, initial diagnosis, or Medicaid eligibility between the two groups. Of the 307 cases, 104 were prescribed medication or physical therapy while 124 received a second visit. One-third of African-Americans received PD treatment compared to Caucasians $(p=0.002)$. There were no differences in the number of return clinic visits. This study highlights the racial disparities in treatment of $\mathrm{PD}$. The authors hypothesized that lack of effective communication from providers to African-American patients with PD may have contributed to the gap in quality of care. It was also unclear, if the lower rate of treatment among African-Americans were from the providers not prescribing, or the patients not accepting treatment.

The study was limited by selection bias, as the patients with PD who applied for Medicaid were typically low income and disabled. It also lacked documentation of the clinical reasoning or decision making that may have contributed to African-Americans not receiving treatments, such as comorbid conditions that make treatment difficult [5]. The authors recommended interventions to address disparity, such as community, patient and physician awareness, as well as developing interventions to reduce inequities in care. However, these were not discussed in detail.

Cheng et al. analyzed disparities of care among veterans with $\mathrm{PD}$ in a retrospective study using ICD-9 code 332.0 from October 1, 2001 to September 30, 2002 followed by further chart review of the same patients, from 1998 to 2004. Medical charts were reviewed by trained nurses blinded to the study hypothesis to confirm the diagnosis of $\mathrm{PD}$ using ten indicator measures of PD care [6]. Out of five hundred seventy-seven cases with ICD-9 code 332.0, four hundred one PD cases were confirmed. Of those cases, twenty-seven were excluded due to lack of information about race or ethnicity. Results showed that physicians were more likely to confirm a diagnosis of PD among Non-Hispanic Whites than non-Whites. Physicians assessed depression equally among non-Hispanic whites and non-Whites, but the non-Hispanic whites were more likely to 
start treatment and receive follow-up. Patients who were offered treatment for depression and refused were included in the initial treatment group. Many providers did not document if they offered treatment for depression among the untreated group.

\subsection{Access and Treatment Disparities}

Dahodwala et al., Cheng et al., and Chan et al. highlighted several ways race affects access to healthcare and treatment. Each of the studies revealed that even after being diagnosed with PD, African-American patients did not receive adequate first line medications, physical therapy, treatment for depression, or referral for deep brain stimulation (DBS) procedure [5] [6] [7].

African-Americans were found to have significantly fewer DBS procedures compared to Whites (unadjusted odds ratio, 0.13 ; 95\% confidence interval, $0.11-0.16 ; \mathrm{P}<0.001$ ) [7]. After adjusting for comorbidities that may contraindicate DBS, African-Americans were eight times less likely to receive DBS treatment. Despite the fact that African-Americans were more likely to receive care from urban hospitals with a higher number of neurologists and neurosurgeons.

The disparities in care also included treatment for Parkinson's disease depression (PDD). Proper treatment of depression is associated with better quality of life. Cheng et al. reported that physicians did not consistently document whether PD patients who were depressed refused either medication or mental health referral. The rates of initial treatment ( $92 \%$ vs. $80 \%, \mathrm{p}=0.04$ ), and follow-up treatment ( $82 \%$ vs $64 \%, \mathrm{p}=0.05$ ) of depression among African-Americans were lower than non-Hispanic whites, despite there being no difference between race and ethnicity in the assessment of depression [6].

Dahodwala et al., reported that African-Americans were four times less likely than whites to receive any PD treatment (odds ratio, 0.24; 95\% confidence interval, 0.09 0.64 ), including physical therapy. This raises concern for communication breakdown among the providers [5]. They also reported a difference in expectations between the populations about normal aging.

\subsection{Perceptions of Normal Aging}

An interesting confounding variable regarding PD epidemiology in different populations is variation in perceptions of normal aging. Dahodwala and colleagues were the first to examine this by comparing the stage of disease at which African-Americans and white PD patients were first diagnosed [8]. By comparing records from the Philadelphia Veterans Affairs Medical Center, they determined that African-American patients initially presented at more advanced stages of disease compared to their white counterparts (Hoehn and Yahr stage, 2.5 vs 2.0). Surprisingly, despite greater motor impairment, the degree of reported disability among African-Americans was half of what whites reported (Unified Parkinson Disease Rating Scale-disability, $40 \%$ vs $81 \%$ ). This striking discovery regarding disease perception provides a plausible, yet likely partial, explanation for the documented discrepancies in PD prevalence between African- 
Americans and Caucasians.

Discrepancies in perceptions of normal motor performance with aging was explored directly again by Dahodwala and colleagues [9]. They generated a novel assessment tool for testing participants' views on Expectations Regarding Movement (ERM) with questions tailored for parkinsonian symptoms. The investigators used this questionnaire to assess 210 senior center residents in Delaware County, Pennsylvania and demonstrated African-Americans held significantly lower expectations for maintenance of mobility compared to whites. However, there was no difference between the groups with respect to expectations of aging in general. Other participant characteristics that significantly improved ERM scores were higher education (more than high school) and better selfreported health (very good or excellent).

Another study investigated the issue of perceptional variation in PD with a twopronged approach in senior communities in Philadelphia [10]. The study first held focus groups in senior centers to obtain a qualitative understanding of differences in knowledge and perspectives on PD amongst whites, African-Americans and ChineseAmericans. In the quantitative arm of the study, Pan and colleagues constructed a questionnaire based on the findings from the 75 focus group participants. The questionnaire was administered to 154 individuals and revealed commonalities and differences between the three racial groups. Two common themes shared across groups were an overall lack of knowledge regarding the disease, and concern over losing independence as a consequence of developing PD. Distrust of the healthcare system and language barriers were identified as unique obstacles to treatment perceived by Africanand Chinese-Americans. Echoing previous studies, African- and Chinese-Americans were both more likely to view parkinsonian symptoms as part of normal aging compared to whites.

These three illustrative studies revealed substantial differences in both expectations for normal aging and perceived degree of disability from parkinsonian symptoms. The work highlights a likely contributing factor in the variance in PD prevalence amongst different racial and ethnic groups. These studies also point to the importance and need for outreach and education regarding PD in the senior population.

\section{Discussion}

Many of the studies revealed the incidence and prevalence of PD are higher amongCaucasians compared to African-Americans. Since the McInerny-Leo et al. literature review many of the study designs have improved. Yet, there are still limitations making it difficult to assess the overall prevalence of PD among African-Americans compared to Caucasians. For example, Willis et al. provided the largest study to-date on PD incidence and prevalence among African-American population compared to Caucasians by using the Unites States health care database. There are several strengths within this study, including the large sample size and serial cross-sectional study design. However, there were a few limitations, including false positives, case clustering and under-diagnosis due to lack of healthcare access, which may have skewed the overall prevalence of PD in 
the African-American population.

Studies analyzing access and treatment disparities revealed a delay in diagnosis and treatment bias [8]. The studies were retrospective, which evaluated ICD-9 codes. Therefore, they could not determine if the patient refused treatment or if the physician did not offer standard treatment making it difficult to determine the cause of these disparities. There were many hypotheses, which included underreporting of symptoms, mistrust of the medical field and lack of effective communication between the patient and physician [10]. African-Americans were also found to have greater disease severity and disability compared to whites likely as a result of delay in diagnosis and treatment [2]. The studies that revealed a treatment bias also did not address ways to prevent this challenging issue.

There were two studies analyzing the misperceptions of motor performance with aging. Pan et al. analyzed expectations of normal aging via mixed-methods, which revealed several barriers to attitudes and knowledge. Understanding these barriers would improve delays in diagnosis and treatment. The study revealed African-Americans and Chinese-Americans perceived parkinsonian symptoms as a part of normal aging. Therefore, many of the symptoms that may seem vague to the patient become the physician's responsibility to educate them and their care providers.

Are physicians asking the right questions to get an accurate history based on racial and cultural differences? Additional training to the initial primary provider and outreach programs, such as increasing patient awareness are warranted.

McInerney-Leo et al., recommended future studies such as, a long-term survey of a population with a similar proportion of Caucasians and African-Americans similar to the door-to-door survey of Parkinson's disease in Copiah County, Mississippi, and Nigeria [11]. Dahodwala et al., also analyzed racial differences in the diagnosis of Parkinson's disease in a cohort study and they came to a similar conclusion. The racial differences in PD incidence were not elucidated by age, sex, income, insurance, geographic variation or healthcare utilization. They concluded that the racial disparities were more likely a result of discrimination or bias and a population-based approach will help determine the root cause of these differences.

These studies support the need for a long population-based study to analyze the true incidence of Parkinson's disease and better understand how various biases can cause under diagnosis and delay in treatment. It will also help with targeted interventions, such as screening surveys, teaching measures to prevent under-reporting of symptoms and under-diagnoses. Understanding the epidemiological constructs of race in PD will allow us to have a better understanding of Parkinson's disease and help us improve care for an underserved population.

\section{Conclusions}

There were twelve studies analyzing the prevalence, incidence, management and treatment of Parkinson's disease among people of color compared with whites. The majority of the studies used databases, such as the United States Medical benefit recipients, the 
Pennsylvania Medicaid claims, and death certificates and medical charts of veterans with Parkinson's disease [3] [5] [6] [12] [13]. Since the McInerney-Leo article, there has been one cross-sectional study at a Movement Disorders center reviewing social and socioeconomic disparities [2].

Studies analyzing access to care and management were retrospective chart reviews. They found a disproportionate number of African-Americans receiving less care, including medical treatment, physical therapy, depression management, and deep brain stimulation. Although the studies did not analyze the causes of the disparities between African Americans and whites, they highlighted a problem with communication and under-reporting [5] [7] [8] [14].

Three studies analyzed the perception and knowledge of PD symptoms in different populations [8] [9] [10]. These studies emphasized the differences between racial and ethnic groups, but did not reveal the root of these perceptions.

The review articles revealed racial disparities in every facet of Parkinson's disease, from knowledge about the disease, to diagnosis, and finally treatment. Racial disparities also existed within the Veterans' Affairs health care system, which should provide an equal standard of care to all veterans. Unfortunately, the studies do not address the causes of these observations and further studies need to be conducted to determine racial. A community-based study has yet to be performed and may deepen our understanding of Parkinson's disease.

\section{References}

[1] McInerney-Leo, A., Gwinn-Hardy, K. and Nussbaum, R.L. (2004) Prevalence of Parkinson's Disease in Populations of African Ancestry: A Review. Journal of the National Medical Association, 96, 974.

[2] Hemming, J.P., Gruber-Baldini, A.L., Anderson, K.E., Fishman, P.S., Reich, S.G., Weiner, W.J. and Shulman, L.M. (2011) Racial and Socioeconomic Disparities in Parkinsonism. Archives of Neurology, 68, 498-503. https://doi.org/10.1001/archneurol.2010.326

[3] Wright Willis, A., Evanoff, B.A., Lian, M., Criswell, S.R. and Racette, B.A. (2010) Geographic and Ethnic Variation in Parkinson Disease: A Population-Based Study of US Medicare Beneficiaries. Neuroepidemiology, 34, 143-151. https://doi.org/10.1159/000275491

[4] Pagano, G., Ferrara, N., Brooks, D.J. and Pavese, N. (2016) Age at Onset and Parkinson Disease Phenotype. Neurology, 86, 1400-1407. https://doi.org/10.1212/WNL.0000000000002461

[5] Dahodwala, N., Xie, M., Noll, E., Siderowf, A. and Mandell, D.S. (2009) Treatment Disparities in Parkinson's Disease. Annals of Neurology, 66, 142-145.

https://doi.org/10.1002/ana.21774

[6] Cheng, E.M., Siderowf, A.D., Swarztrauber, K., Lee, M., Vassar, S., Jacob, E., Eisa, M.S. and Vickrey, B.G. (2008) Disparities of Care in Veterens with Parkinson's Disease. Parkinsonism \& Related Disorders, 14, 8-14. https://doi.org/10.1016/j.parkreldis.2007.05.001

[7] Chan, A.K., McGovern, R.A., Brown, L.T., Sheehy, J.P., Zacharia, B.E., Mikell, C.B., Bruce, S.S., Ford, B. and McKhann, G.M. (2014) Disparities in Access to Deep Brain Stimulation Surgery for Parkinson Disease: Interaction between African American Race and Medicaid use. JAMA Neurology, 71, 291-299. https://doi.org/10.1001/jamaneurol.2013.5798 
[8] Dahodwala, N., Karlawish, J., Siderowf, A., Duda, J.E. and Mandell, D.S. (2011) Delayed Parkinson's Disease Diagnosis among African-Americans: The Role of Reporting of Disability. Neuroepidemiology, 36, 150-154. https://doi.org/10.1159/000324935

[9] Dahodwala, N., Karlawish, J., Shea, J.A., Zubritsky, C., Stern, M. and Mandell, D.S. (2012) Validation of an Instrument to Measure Older Adults' Expectations Regarding Movement (ERM). PloS One, 7, Article ID: e43854. https://doi.org/10.1371/journal.pone.0043854

[10] Pan, S., Stutzbach, J., Reichwein, S., Lee, B.K. and Dahodwala, N. (2014) Knowledge and Attitudes About Parkinson's Disease Among a Diverse Group of Older Adults. Journal of cross-Cultural Gerontology, 29, 339-352. https://doi.org/10.1007/s10823-014-9233-x

[11] Schoenberg, B.S., Osuntokun, B.O., Adeuja, A.O., Bademosi,O., Nottidge, V., Anderson, D.W. and Haerer, A.F. (1988) Comparison of the Prevalence of Parkinson's Disease in Black Populations in the Rural United States and in Rural Nigeria Door-to-Door Community Studies. Neurology, 38, 645. https://doi.org/10.1212/WNL.38.4.645

[12] Pressley, J.C., Tang, M.X., Marder, K., Cote, L.J. and Mayeux, R. (2005) Disparities in the Recording of Parkinson's Disease on Death Certificates. Movement Disorders, 20, 315-321. https://doi.org/10.1002/mds.20339

[13] Dahodwala, N., Siderowf, A., Xie, M., Noll, E., Stern, M. and Mandell, D.S. (2009) Racial Differences in the Diagnosis of Parkinson's Disease. Movement Disorders, 24, 1200-1205. https://doi.org/10.1002/mds.22557

[14] Yacoubian, T.A., Howard, G., Kissela, B., Sands, C.D. and Standaert, D.G. (2009) Racial Differences in Parkinson's Disease Medication Use in the Reasons for Geographic and Racial Differences in Stroke Cohort: A Cross-Sectional Study. Neuroepidemiology, 33, 329334. https://doi.org/10.1159/000254568

[15] Tilley, B.C., Mainous, A.G., Elm, J.J., Pickelsimer, E., Soderstrom, L.H., Ford, M.E., Diaz, V.A., Siminoff, L.A., Burau, K. and Smith, D.W. (2012) A Randomized Recruitment Intervention Trial in Parkinson's Disease to Increase Participant Diversity: Early Stopping for Lack of Efficacy. Clinical Trials, 9, 188-197. https://doi.org/10.1177/1740774512436881

Submit or recommend next manuscript to SCIRP and we will provide best service for you:

Accepting pre-submission inquiries through Email, Facebook, LinkedIn, Twitter, etc.

A wide selection of journals (inclusive of 9 subjects, more than 200 journals)

Providing 24-hour high-quality service

User-friendly online submission system

Fair and swift peer-review system

Efficient typesetting and proofreading procedure

Display of the result of downloads and visits, as well as the number of cited articles

Maximum dissemination of your research work

Submit your manuscript at: http://papersubmission.scirp.org/

Or contact apd@scirp.org 